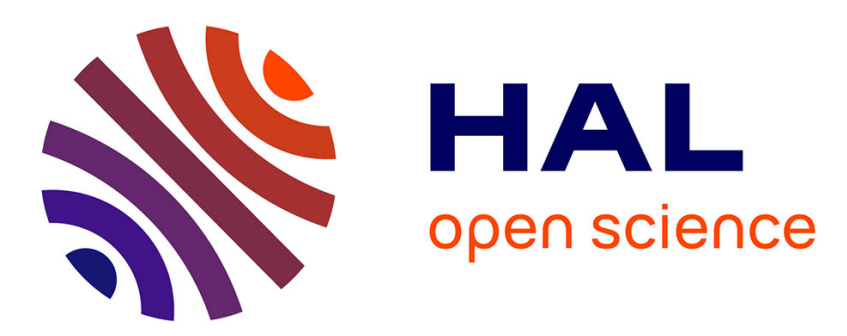

\title{
Collision avoidance between two walkers: Role-dependent strategies
}

\author{
Anne-Hélène Olivier, Antoine Marin, Armel Crétual, Alain Berthoz, Julien \\ Pettré
}

\section{To cite this version:}

Anne-Hélène Olivier, Antoine Marin, Armel Crétual, Alain Berthoz, Julien Pettré. Collision avoidance between two walkers: Role-dependent strategies. Gait \& Posture, 2013, 10.1016/j.gaitpost.2013.03.017 . hal-00821854

\section{HAL Id: hal-00821854 https://hal.inria.fr/hal-00821854}

Submitted on 13 May 2013

HAL is a multi-disciplinary open access archive for the deposit and dissemination of scientific research documents, whether they are published or not. The documents may come from teaching and research institutions in France or abroad, or from public or private research centers.
L'archive ouverte pluridisciplinaire HAL, est destinée au dépôt et à la diffusion de documents scientifiques de niveau recherche, publiés ou non, émanant des établissements d'enseignement et de recherche français ou étrangers, des laboratoires publics ou privés. 


\section{Collision avoidance between two walkers: role- dependent strategies}

Anne-Hélène Olivier ${ }^{1,2}$, Antoine Marin ${ }^{1}$, Armel Crétual ${ }^{1,2,}$ Alain Berthoz ${ }^{3}$ and Julien Pettré2

1Laboratoire Mouvement Sport Santé (M2S), UFRAPS, Université Rennes 2-ENS Cachan, Avenue Charles Tillon, CS24414, 35044 Rennes, France.

2 INRIA, Centre de Rennes Bretagne Atlantique, Campus Universitaire de Beaulieu, 263 avenue du Général Leclerc, 35042 Rennes, France.

3 Laboratoire de Physiologie de la Perception et de l'Action (LPPA), Collège de France, 11 place Marcelin Berthelot, 75231 Paris, France.

Phone: +33299847138

Email address:

anne-helene.olivier@inria.fr, antoine.marin@univ-rennes2.fr, armel.cretual@univ-rennes2.fr, alain.berthoz@college-de-france.fr, julien.pettre@inria.fr

Acknowledgements:

The project Tango acknowledges the financial support of the Future and Emerging Technologies (FET) Programme within the Seventh Framework Programme for Research of the European Commission, under FET-Open grant number: 249858". This work was also funded by the french ANR-PsiRob 2007 Locanthrope Project (ANR-07-ROBO-0007). 


\section{Abstract}

This paper studies strategies for collision avoidance between two persons walking along crossing trajectories. It has been previously demonstrated that walkers are able to anticipate the risk of future collision and to react accordingly. The avoidance task has been described as a mutual control of the future distance of closest approach, MPD (i.e., Mininum Predicted Distance). In this paper, we studied the role of each walker in the task of controlling MPD. A specific question was: does the walker giving way $\left(2^{\text {nd }}\right.$ at the crossing) and the one passing first set similar and coordinated strategies? To answer this question, we inspected the effect of motion adaptations on the future distance of closest approach. This analysis is relevant in the case of collision avoidance because subtle anticipatory behaviors or large last moment adaptations can finally yield the same result upon the final crossing distance. Results showed that collision avoidance is performed collaboratively and the crossing order impacts both the contribution and the strategies used: the participant giving way contributes more than the one passing first to avoid the collision. Both walkers reorient their path but the participant giving way also adapts his speed. Future work is planned to investigate the influence of crossing angle and TTC on adaptations as well as new types of interactions, such as intercepting or meeting tasks. 


\section{Introduction}

Collision-free walking requires avoiding static and moving obstacles and, more specifically, other walkers. Collision avoidance can be described as a kinematic motion control problem with two main aspects: the visual information taken and the motion adaptations performed by walkers. Previous studies [1,2] focused on the nature of visual information taken by walkers to answer two questions: is there a risk of future collision, and when may collision occur? Cutting et al. [1] showed that by-pass or collision can be predicted up to 10 seconds before contact based on gaze movement angle. When a future collision is detected, walkers can estimate the time-to-contact (TTC). TTC can be indicated by the optical variable tau $[3,4,5]$, by the binocular disparity alone [6] or combined with retinal information [7]. TTC estimation gets more accurate as the contact time gets closer [8].

Collision avoidance is also related to the notion of personal space, defined as an area around walkers which is maintained free thanks to some collision avoidance adaptations [9]. Stepping over $[10,11,12]$ or circumventing $[13,14,15]$ of static obstacle(s) was studied. Some work focused on passive moving obstacles, such as a mannequin mounted on a rail [9,16,17]. Various contextdependent strategies were observed. When walking participants meet mannequins following a $45^{\circ}$ colliding paths, they adapt their motion both in the antero-posterior and medio-lateral planes [9]. When a participant and a mannequin are walking face-to-face, a 2-step avoidance strategy is observed: first, a change in heading and second, an adjustment of walking speed [17]. Interestingly, the initiation of adaptations is performed at a constant distance from the obstacle whatever the obstacle velocity [17]. Moreover, obstacle velocity influences the lateral rate of change of the walker's trajectory [16]: the slower the velocity, the lower the lateral rate of change. Finally, interactions between a walker and the environment were modeled as coupled dynamical systems 
$[18,19,20]$. Heading is adapted according to the distance and the angle to stationary goals and obstacles.

However, few studies considered interactions between two human walkers [21,22,23]. Ducourant et al. [21] focused on leader-follower interactions between two participants walking back and forth, face to face. Van Basten et al. [22] investigated the effect of gender and height on face-to-face situations of avoidance. More recently, the case of two humans walking along $90^{\circ}$ colliding paths was studied [23]. Analysis was based on the Minimal Predicted Distance (MPD), which is the future distance of closest approach between two walkers if they continue walking straight and at constant speed: it is deduced by linearly extrapolating future trajectories from each walker's current position, heading and speed. The change of MPD in time showed that walkers adapt their trajectories only when MPD is initially low $(<1 \mathrm{~m})$. This shows the ability to predict future risks of collision and to react accordingly. Also, collision avoidance can be described as the task of mutually controlling MPD. Experimental observation of the temporal evolution of MPD(t) showed that collision avoidance presents 3 successive phases: the observation phase (low MPD) is followed by a reaction phase (MPD is increased to an acceptable value) and a regulation phase (the acceptable value is maintained). When the regulation phase starts, the avoidance is solved and then carried out: avoidance is performed with anticipation. The change of MPD in time is necessarily due to motion adaptation (non-linear trajectories), but how $\operatorname{MPD}(\mathrm{t})$ is individually controlled still needs analysis.

The purpose of the current study was to analyze collision avoidance adaptations between two walkers. In addition we explored the strategy (speed and/or heading adaptations) set by walkers to avoid future collisions. When do these adaptations take place? Is this avoidance task solved collaboratively? 
We addressed these questions from several new perspectives. First, adaptations were quantified in terms of their effect on the future crossing distance. Second, we inspected the effect of the participants' crossing order (i.e., who is first, who gives way) on their individual avoidance strategy. Indeed, the participant giving way has the participant passing first in front of him/her, and the participant passing first has the second one to his/her side or behind him/her (Fig.1). This asymmetric configuration leads us to emphasize asymmetric adaptations for collision avoidance. Indeed, as suggested by Gérin-Lajoie et al. [9], personal space has an elliptic shape. Collision risk should be perceived as being higher when the walker to avoid is in front compared to the side and therefore it should induce different avoidance adaptations

\section{Materials and Methods}

\section{Participants}

Thirty participants (11 women and 19 men) volunteered for this experiment. They were 26.1 years old $( \pm 6.9)$ and $1.74 \mathrm{~m}$ tall $( \pm 0.09)$. They had no known vestibular, neurological or musculo-skeletal pathologies which would affect their locomotion. They had normal or corrected to normal vision. Participants gave written and informed consent before their inclusion and the study conformed to the Declaration of Helsinki.

\section{Experimental protocol and apparatus}

We asked participants to go from one corner to the opposite corner of a $15 \times 15 \mathrm{~m}$ square experimental area (Fig.2A). There were five groups of six participants. Each participant interacted with each of the five other ones. Each participant performed 30 trials, (i.e., 6 interactions with each of the other participants). Therefore, the total number of trials performed, accounting for all paired 
interactions, was 450. However, 30 trials were suppressed because of motion reconstruction problems. Participants had neither instruction nor restriction about their gait speed and path. We synchronized their start signals to induce risks of collision. The presence of occluding walls prevented participants from seeing each other before reaching their comfort speeds. At visual contact, participants were about $6 \mathrm{~m}$ from the center of the area. This study focused on a subset of 260 trials for which an actual risk of collision was measured: risk of collision is true when the Minimal Predicted Distance (MPD) is smaller than one meter at visual contact as defined by Olivier and colleagues [23].

\section{Analysis}

3D kinematic data were recorded using the Vicon-MX system $(120 \mathrm{~Hz})$, reconstruction was performed using Vicon-IQ (Oxford Metrics®) and computations using Matlab (Mathworks®). We approximated participants' position by the middle of their shoulders (acromions). In the aim of computing MPD (see below) and to correctly estimate current speed and orientation, we filtered the stepping oscillations by applying a Butterworth low-pass filter ( $3 \mathrm{rd}$ order, dual pass, $0.5 \mathrm{~Hz}$ cutoff frequency). Velocity was computed as the discrete time derivative of the mid-shoulders position in the horizontal plane.

We computed tsee, tcross and dmin from experimental data as defined in [23] (Fig.2A). 1) tsee is the time-value when participants are able to see each other, with respect to occluding walls geometry. 2) dmin is the actual minimum distance measured between participants. 3) tcross is the time-value when the distance dmin is reached by participants. 


\section{Minimal Predicted Distance}

We computed Minimal Predicted Distance (MPD) as defined in [23]. MPD(t) is, at time t, the prediction of the future distance of closest approach between participants if they do not perform adaptation and keep their velocity vector constant.

MPD(tsee) is the predicted distance of closest approach at time tsee, when participants are able to have visual contact. MPD(tsee) varied in experimental data due to the variability in reaction time to the start signal and comfort speed among participants. Olivier and colleagues [23] showed that motion adaptations are observed during interaction only when MPD(tsee) was low (smaller than 1 meter). We selected trials in which MPD(tsee) was smaller than 1 meter to focus our study on data actually containing motion adaptations.

\section{Temporal segmentation}

It was shown that collision avoidance can be decomposed into 3 successive phases: observation, reaction and regulation [23]. Our study focused on adaptation strategy which occurs in the reaction phase. Thus, we considered the central portion of data where $80 \%$ of total MPD variation is performed (from $10 \%$ to $90 \%$ of total variation) as shown in Fig.2B. Then, for each trial we performed a temporal normalization on this phase to enable comparisons. This time window will be called "normalized reaction phase".

\section{Collision avoidance adaptations}

We distinctly evaluated motion adaptations performed by the walker passing first (participant \#1) from those of the participant giving way (participant\#2). Collision avoidance was analyzed both with respect to speed (s) and orientation $(\theta)$ adaptations on the normalized reaction phase. In this study, we took more interest in the effect of adaptations on the situation than in the adaptations themselves. 
These effects are revealed by computing the partial derivatives of $\mathrm{MPD}(\mathrm{t})$ with respect to motion adaptations performed by participants. Indeed this shows how much $\operatorname{MPD}(t)$ is adapted when the orientation or speed of participant \#1 or \#2 varies.

We noted at time $\mathrm{t} \theta_{1}$ and $s_{1}$ (resp. $\theta_{2}$ and $s_{2}$ ) the instantaneous orientation and speed of participant\#1 (resp. \#2) and $X=(a, b)$ the relative position of participant\#2 with respect to \#1. Then, we have:

$$
\operatorname{MPD}(t)=f\left(a, b, \theta_{1}, s_{1}, \theta_{2}, s_{2}\right)
$$

For any parameter $p$ among the $6 f$ depended on, the instantaneous individual effect of $p$ is:

$$
\varepsilon_{p}=\frac{\partial f}{\partial p} \times d p
$$

The instantaneous total effect $\nabla f$ on MPD is therefore:

$$
\nabla f=\varepsilon_{a}+\varepsilon_{b}+\varepsilon_{\theta_{1}}+\varepsilon_{\theta_{2}}+\varepsilon_{s_{1}}+\varepsilon_{s_{2}}
$$

Because of the obvious link between position variation and velocity, it can be shown that (see supplementary material for details):

$$
\varepsilon_{a}+\varepsilon_{b}=0
$$

Therefore, we have:

$$
\nabla f=\varepsilon_{\theta_{1}}+\varepsilon_{\theta_{2}}+\varepsilon_{s_{1}}+\varepsilon_{s_{2}}
$$


These instantaneous effects are illustrated in Fig.2C. We can evaluate the cumulative contribution of each participant with respect to each type of adaptation by integrating the instantaneous effect in time (Fig.2D).

$$
\begin{aligned}
& C \varepsilon_{\theta_{1}}=\int_{0}^{t} \frac{\partial f}{\partial \theta_{1}} \times \frac{d \theta_{1}}{d t} \times d t \\
& C \varepsilon_{\theta_{2}}=\int_{0}^{t} \frac{\partial f}{\partial \theta_{2}} \times \frac{d \theta_{2}}{d t} \times d t \\
& C \varepsilon_{s_{1}}=\int_{0}^{t} \frac{\partial f}{\partial s_{1}} \times \frac{d s_{1}}{d t} \times d t \\
& C \varepsilon_{s_{2}}=\int_{0}^{t} \frac{\partial f}{\partial s_{2}} \times \frac{d s_{2}}{d t} \times d t
\end{aligned}
$$

The cumulative total effect $\nabla C f$ on MPD is therefore:

$$
\nabla C f=C \varepsilon_{\theta_{1}}+C \varepsilon_{\theta_{2}}+C \varepsilon_{s_{1}}+C \varepsilon_{s_{2}}
$$

Finally, we can deduce the contribution in percentage of re-orientations $\% C \varepsilon_{\theta_{1}}, \% C \varepsilon_{\theta_{2}}$ or speed adaptations $\% C \varepsilon_{s_{1}}, \% C \varepsilon_{s_{2}}$ and of each participant, $\% C \varepsilon_{1}, \% C \varepsilon_{2}$ on MPD. 


$$
C \varepsilon_{\theta_{1}}=\frac{C \varepsilon_{\theta_{1}}}{\nabla C f} \times 100
$$

$$
C \varepsilon_{\theta_{2}}=\frac{C \varepsilon_{\theta_{2}}}{\nabla C f} \times 100
$$

$$
C \varepsilon_{s_{1}}=\frac{C \varepsilon_{s_{1}}}{\nabla C f} \times 100
$$

$$
C \varepsilon_{s_{2}}=\frac{C \varepsilon_{s_{2}}}{\nabla C f} \times 100
$$

$$
C \varepsilon_{1}=\frac{C \varepsilon_{\theta_{1}}+C \varepsilon_{\varepsilon_{1}}}{\nabla C f} \times 100
$$

$$
C \varepsilon_{2}=\frac{C \varepsilon_{\theta_{2}}+C \varepsilon_{{\mho_{2}}_{2}}}{\nabla C f} \times 100
$$

\section{Statistics}

Results were presented with mean plus or minus Standard Error of the Mean. All effects were reported at $\mathrm{p}<0.05$. Normality distribution was assessed using the Kolmogorov-Smirnov test. We compared participant\#1 and participant\#2 speeds at time tsee using a paired t-test. To compare the contribution of each participant on the collision avoidance task, we performed Wilcoxon signedrank tests for each of the 101 instants of the normalized reaction phase. We performed the same 10 
tests to compare instantaneous effects in speed and orientation for each participant. To evaluate the effect of avoidance strategies (speed and orientation of each participant) and their respective differences, Friedman's Anova and post-hoc Wilcoxon signed-rank tests were performed on the contribution of each strategy in collision avoidance at each instant of the normalized reaction phase. A Bonferroni correction was applied and so all effects are reported at a 0.0083 level of significance $(0.05 / 6)$ to adapt critical value for significance for these Wilcoxon signed-rank post-hoc tests.

\section{Results}

We analyzed the 260-trial subset (for which $\operatorname{MPD}($ tsee $)<1 \mathrm{~m}$ ) which exhibits actual collision avoidance adaptations. The "normalized reaction phase" started $3 \mathrm{~s}( \pm 0.05)$ before tcross, and ended 1.6s $( \pm 0.04)$ before tcross and lasted $1.4 \mathrm{~s}( \pm 0.04)$. From a global point of view, MPD(t) increases linearly during the reaction phase $\left(\mathrm{R}^{2}=0.99, \mathrm{p}<0.001\right)$ from an average of $0.38 \mathrm{~m}$ to $0.86 \mathrm{~m}$. There was no significant difference between the speeds of participant\#1 $(1.58 \mathrm{~m} / \mathrm{s} \pm 0.01)$ and \#2 $(1.57 \mathrm{~m} / \mathrm{s} \pm 0.01)$ at $\mathrm{tsee}(\mathrm{t}=1.13, \mathrm{df}=259, \mathrm{p}=0.258)$. Moreover, there was almost no inversion in the role of each walker: participant\#1 (resp. \#2) at MPD(tsee) is participant\#1 (resp. \#2) at the crossing point.

Collision avoidance was mutually performed by both participants (Fig.3). Nevertheless, Wilcoxon signed-rank tests computed for each instant of the reaction phase (101 tests) indicated that the contribution of participant\#2 to avoid a collision is always more important than the one of participant\#1 (for all tests $\mathrm{p}<0.001$ ). On average, these contributions to increase MPD(t) were $56.8 \%( \pm 0.17)$ and $43.2 \%( \pm 0.17)$ respectively for participants\#2 and \#1.

Figure 4 illustrates the contribution of each participant's strategy to increase MPD(t) during the reaction phase. All of the 101 Friedman tests indicated an influence of the strategy $(p<0.001)$. Posthoc Wilcoxon signed-rank tests showed 3 successive phases in the distribution of these strategy 
contributions. First, from $0 \%$ to $47 \%$ of the normalized reaction phase, the increasing of $\operatorname{MPD}(\mathrm{t})$ was due, in a descending order of contribution, to speed adaptation of participant\#2, to orientation adaptation of both participants and to speed adaptation of participant\#1. Second, from $48 \%$ to $67 \%$, speed adaptation of participant\#2 had similar contribution than orientation of participant\#1 which was similar than the contribution of orientation of participant\#2; speed adaptation of participant\#1 had the lower contribution. Third, from $68 \%$ to $100 \%$ all strategies had similar contributions on collision avoidance, except speed adaptation of participant\#1 which was lower.

We investigated the temporal sequence of motion adaptations for each participant by analyzing instantaneous effects of speed and orientation adaptations on MPD(t) (Fig.5). Wilcoxon signed-rank tests showed that instantaneous adaptation in speed for participant\#1 was always lower than the one in orientation. For participant $\# 2$, from $0 \%$ to $43 \%$, the instantaneous adaptation in speed was greater than the one in orientation. Then, from $44 \%$ to $64 \%$, there was no significant differences between these adaptations ( $>0.05)$. Last, from $65 \%$ to $100 \%$, the instantaneous adaptation in speed was smaller than the one in orientation.

\section{Discussion}

This study provides new insight to collision avoidance between two walkers. Results showed that this task is performed collaboratively and that the crossing order impacts both the contribution and the strategies used.

\section{Collaborative but role-dependent behavior}

A first interesting result is that the $\operatorname{MPD}(\mathrm{t})$ is constantly growing: on average, each walker's effect is always positive (Fig.5) and they collaboratively solve the interaction. By integrating 
instantaneous effects, we evaluated each walker's contribution to the avoidance task. We found a role-dependent behavior (Fig.3). The walker giving way (\#2) contributes more to the avoidance than the one passing first (\#1). We relate this observation to the asymmetry induced by the shape of personal space described by Gérin-Lajoie et al. [9] (see Fig. 1). This asymmetry could also be explained by the difference in the visual input each walker has: obviously, perceiving someone passing in front of oneself is very different to the opposite situation.

\section{Anticipatory versus on line avoidance planning}

A second result is that there was almost no inversion in the role of each walker: a definitive and tacit assignment seems to occur at the very beginning of the reaction phase. In other words, if one of the two walkers is slightly in advance compared to the other one and is more likely to pass first, he will take and keep this role. This role decision making process seems to be resulting from a planning process, similarly to [9].

Nevertheless, we also have arguments in favor of on-line control. First, MPD is mutually adapted to final values at the end of the reaction phase: this would be impossible from independent and planned adaptations. Note that the bearing angle variation [1] can reveal potential roles: an approaching obstacle which is likely to pass in front or behind has a bearing angle which is respectively converging or diverging to the gaze axis.

\section{Reorientation versus speed adaptation of the trajectory}

Walkers' roles not only influenced their respective contributions, but also: i) the type of adaptations and ii) the proportion of these adaptations. Results showed that speed adaptation was preferred by the participant\#2 during the first half of the reaction phase (above 35\% contribution), re-orientation was performed by participants \#1 and \#2 (25\% each), and speed adaptation by participant\#1 (only $15 \%)$. In the second half of the reaction phase, strategies had similar contributions (precisely, speed 
adaptation of participant\#1 which was lower). Instantaneously, the effect of speed change for participant\#1 was always lower than for re-orientation: this walker gave priority to a change in heading. It would be interesting to investigate if this choice takes into account a minimum energy criterion in which strategies can be weighted according to their respective cost. Concerning participant\#2, he/she preferred decreasing speed during the first half of the reaction phase. In the second half, a change of heading is used as well. This result can be related to Gérin-Lajoie et al. [9] in spite of the fact that we considered two walkers instead of using a mannequin: a general decrease of the walking speed and a mediolateral deviation of the locomotor trajectory were shown.

\section{Limitations}

We focused on the role of walkers in the interaction. We analyzed a specific case study at the global level and provided a first insight into interactions between walkers. Nevertheless, we did not consider inter- or intra-individual factors such as for example gender, sociological parameters, psychological parameters (emotions) or physical parameters (body size, walking speed...). These factors may change both the importance and the nature of avoidance strategies. As a result, future work is required to complete the analysis and to propose general behavioral strategies for collision avoidance.

In conclusion, we focused on collision avoidance between two walkers. We considered a typical situation and demonstrated that such an interaction is solved collaboratively, but that the nature and quantity of adaptations differ according to the role of the walker in the crossing. More generally, we proposed a novel framework to analyze this interaction, based on a relevant metric to characterize the situation of an interaction between two walkers [23]. Instead of directly measuring the adaptations, defined as a deviation from straight-walking behavior [9,16,17], we inspected their

effects. We plan to explore two research directions. The first is to study the effects of angle and 
TTC on avoidance adaptations: modulation in time of anticipation and modulation of strategy would be explored based on the proposed framework, and would complete existing results [18, 24]. The second is to extend our framework to new types of interactions, such as leader-follower interactions, intercepting or meeting tasks.

\section{Conflict of interest statement}

None of the authors have any financial and personal relationships with other people or organizations that could inappropriately influence their work.

\section{References}

[1] Cutting J, Vishton P, Braren P. How we avoid collisions with stationary and moving obstacles. Psychological Review 1995; 102: 627-651.

[2] Regan D, Gray R. Visually guided collision avoidance and collision achievement. Trends in Cognitive Sciences 2000; 4: 99-107.

[3] Lee DN. A theory of visual control of braking based on information about time-to-collision. Perception 1976; 5: 437-459.

[4] Kim NG, Turvey MT, Carello V. Optical information about the severity of upcoming contacts. Journal of Experimental Psychology: Human Perception and Performance 1993; 19: 179-193.

[5] Fajen BR Calibration, information, and control strategies for braking to avoid a collision. Journal of Experimental Psychology: Human Perception and Performance 2005; 31: 480-501.

[6] Gray R, Regan D. Accuracy of estimating time to collision using binocular and monocular information. Vision Research 1998; 38: 499-512. 
[7] Rushton SK, Wann JP. Weighted combination of size and disparity: A computational model for timing a ball catch. Nature Neuroscience 1999; 2: 186-190.

[8] Salversberg GJ, Whiting HT, Bootsma RJ. Grasping tau. Journal of Experimental Psychology: Human Perception and Performance 1991; 17: 315-322.

[9] Gérin-Lajoie M, Richards C, McFadyen B. The negociation of stationary and moving obstructions during walking: anticipatory locomotor adaptations and preservation of personal space. Motor Control 2005; 9: 242-269.

[10] Mohagheghi A, Moraes R, Patla A. The effects of distant and online visual information on the control of approach phase and step over an obstacle during locomotion. Experimental Brain Research 2004; 155: 459-468.

[11] Patla A, Rietdyk S, Martin C, Prentice S. Locomotor patterns of the leading and the trailing limbs as solid and fragile obstacles are stepped over: some insights into the role of vision during locomotion. Journal of Motor Behavior 1996; 28: 35-47.

[12] Rhea CK, Rietdyk S. Influence of an unexpected perturbation on adaptive gait behavior. Gait and Posture 2011; 34: 439-441.

[13] Jansen SE, Toet A, Werkhoven PJ. Human locomotion through a multiple obstacle environment: strategy changes as a result of visual field limitation. Experimental Brain Research 2011; 212: 449-456.

[14] Vallis LA, McFadyen BJ. Locomotor adjustments for circumvention of an obstacle in the travel path. Experimental Brain Research 2003; 152: 409-414.

[15] Vallis LA, McFadyen BJ . Children use different anticipatory control strategies than adults to circumvent an obstacle in the travel path. Experimental Brain Research 2005; 167: 119-127.

[16] Cinelli ME, Patla AE. Travel path conditions dictate the manner in which individuals avoid collisions. Gait and Posture 2007; 26: 186-193. 
[17] Cinelli ME, Patla AE. Locomotor avoidance behaviours during a visually guided task involving an approaching object. Gait and Posture 2008; 28: 596-601.

[18] Fajen BR, Warren WH. Behavioral dynamics of steering, obstacle avoidance and route selection. Journal of Experimental Psychology: Human Perception and Performance 2003; 29: 343362.

[19] Warren WH, Di S, Fajen BR. Behavioral dynamics of avoiding a moving obstacle. Journal of Vision 2003; 3: 134.

[20] Cohen JA, Bruggeman H, Warren WH. Switching behavior in moving obstacle avoidance. Journal of Vision 2005; 5: 312.

[21] Ducourant T, Vieilledent S, Kerlirzin Y, Berthoz A. Timing and distance characteristics of interpersonal coordination during locomotion. Neuroscience Letters 2005; 389(1): 6-11.

[22] van Basten BJ, Jansen SE, Karamouzas I. Exploiting motion capture to enhance avoidance behaviour in games. Lecture Notes in Computer Science 2009; 5884: 29-40.

[23] Olivier AH, Marin A, Crétual A, Pettré. Minimal predicted distance: A common metric for collision avoidance during pairwise interactions between walkers. Gait and Posture 2012; 36(3): 399-404.

[24] Manser MP, Hancock PA. Influence of approach angle on estimates of time-to-contact. Ecological Psychology 1996; 8(1): 71-99. 


\section{Captions to illustrations}

Fig.1: Personal space configurations while crossing by extension of the results of Gérin-Lajoie et al. [9] on the elliptic shape of personal space.

Fig.2: A) Experimental setup. 2 participants (P1 and P2) stood at the corners of the area (15x15m) and were synchronously given a start signal. Their task was to walk to the opposite corner. They implicitly started an interaction at tsee (they first can see each other) to avoid any collision until tcross (participants cross each other: the distance between them is minimal (dmin)). B) Corresponding Minimal Predicted Distance (MPD) between the time they can see each other (tsee) and the time they cross (tcross). All analyses focused on the reaction phase. C) Instantaneous effects of avoidance strategies (speed and orientation for both participants) on MPD(t) during the normalized reaction phase. D) Corresponding cumulative effects of avoidance strategies on MPD(t).

Fig.3: Contribution (mean \pm SEM) of each participant on MPD variations during the reaction phase. Both participants increased MPD but participant\#2 provided more effort than participant\#1 to avoid a collision.

Fig.4: Contribution (mean \pm SEM) of each strategy on MPD variations during the reaction phase and statistical significant differences between the contributions (from post-hoc Wilcoxon signed-rank tests).

Fig.5: Instantaneous effect (mean \pm SEM) of each strategy on $\operatorname{MPD}(\mathrm{t})$ over the normalized reaction phase. 

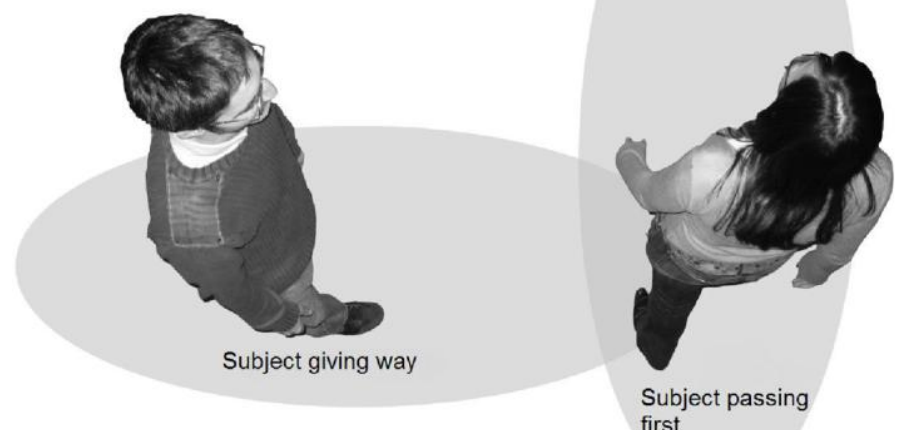

first

19 

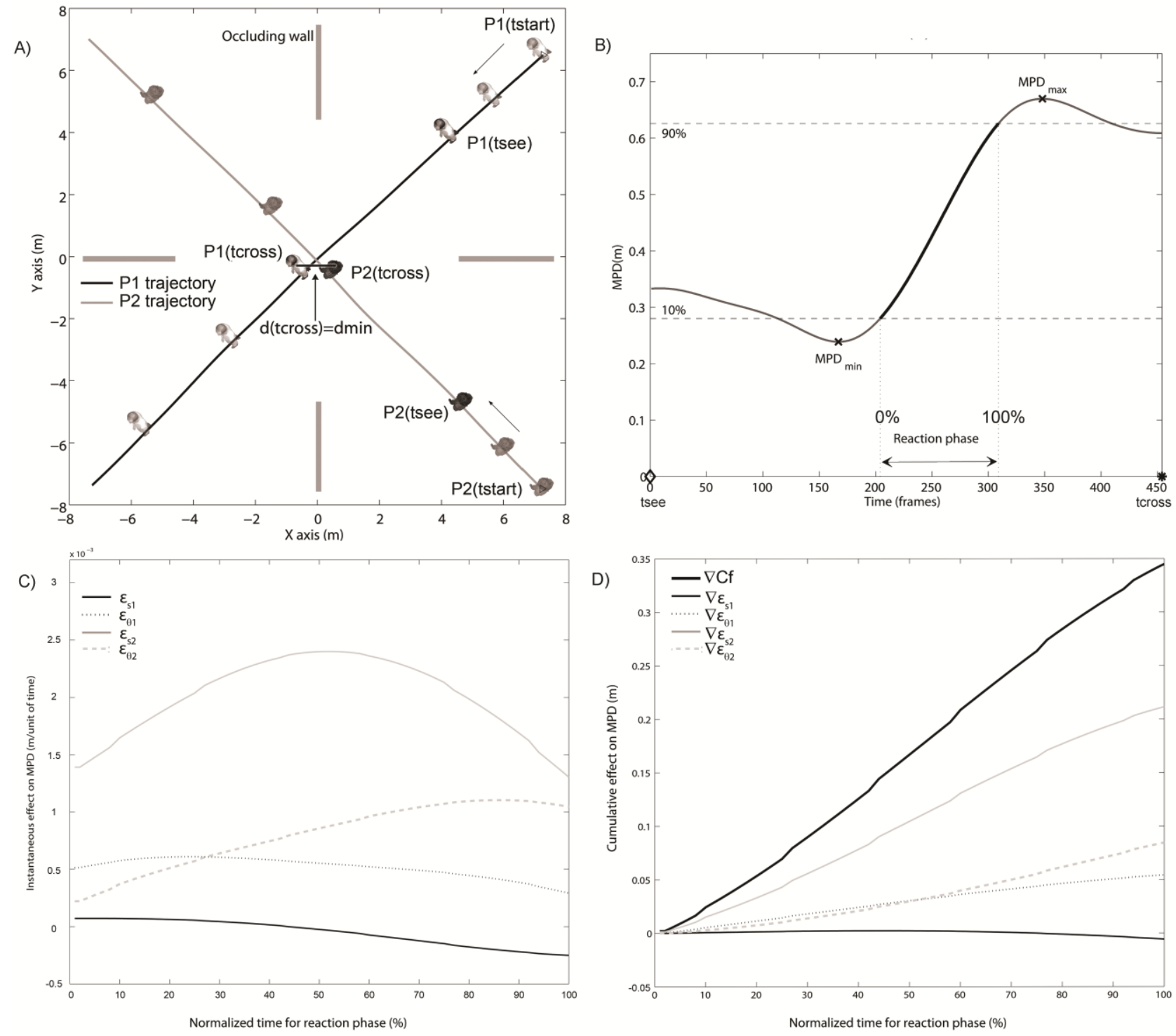


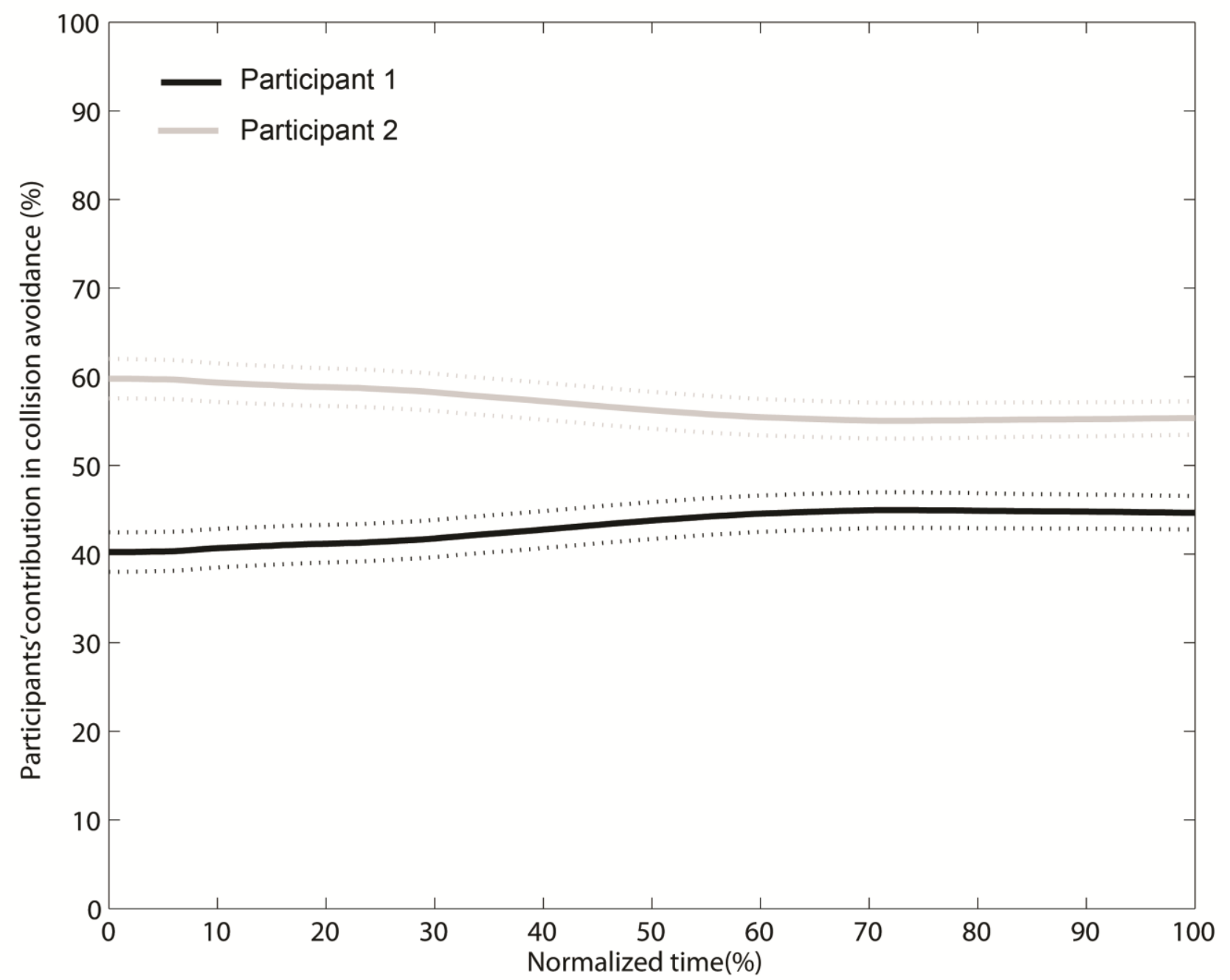




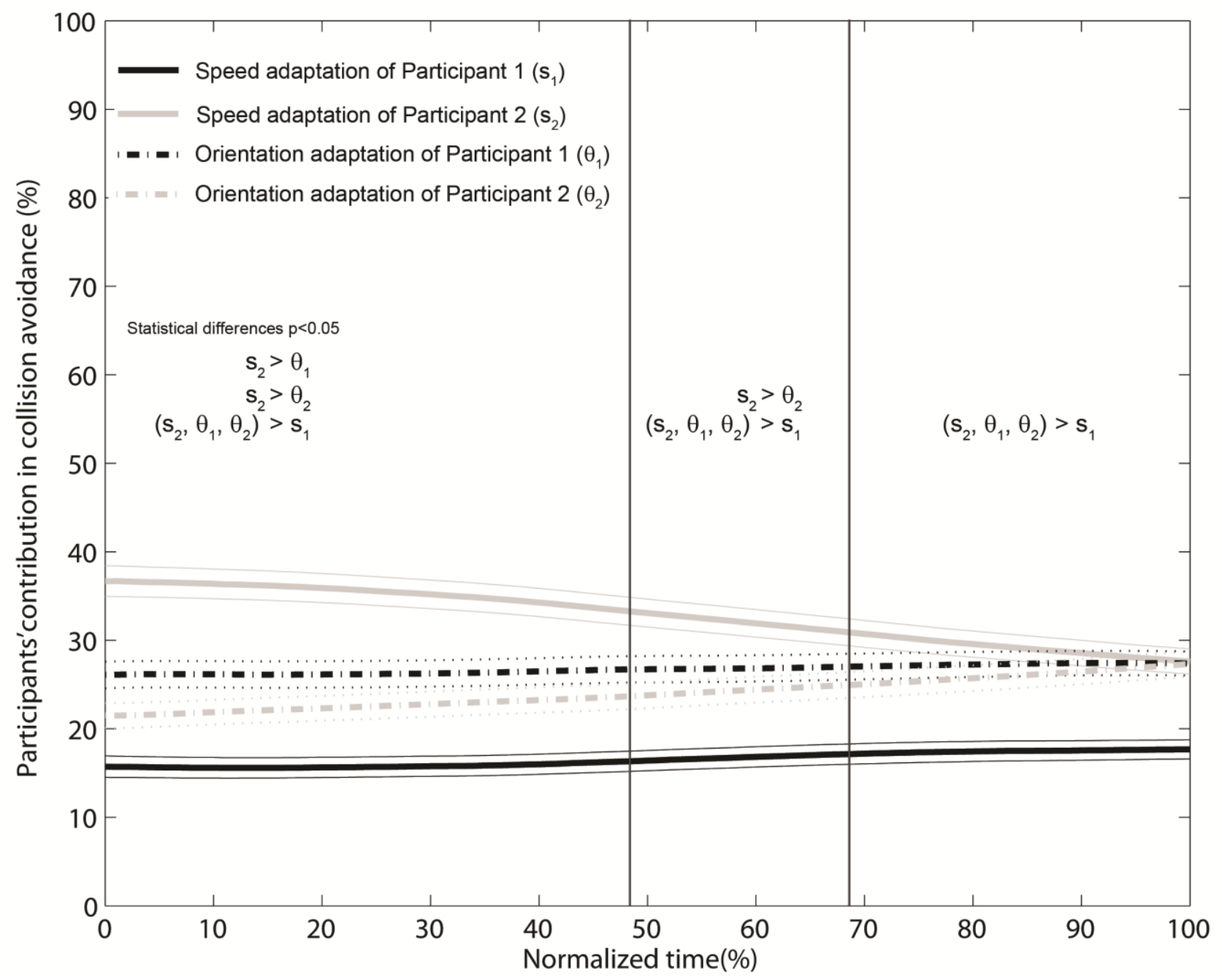




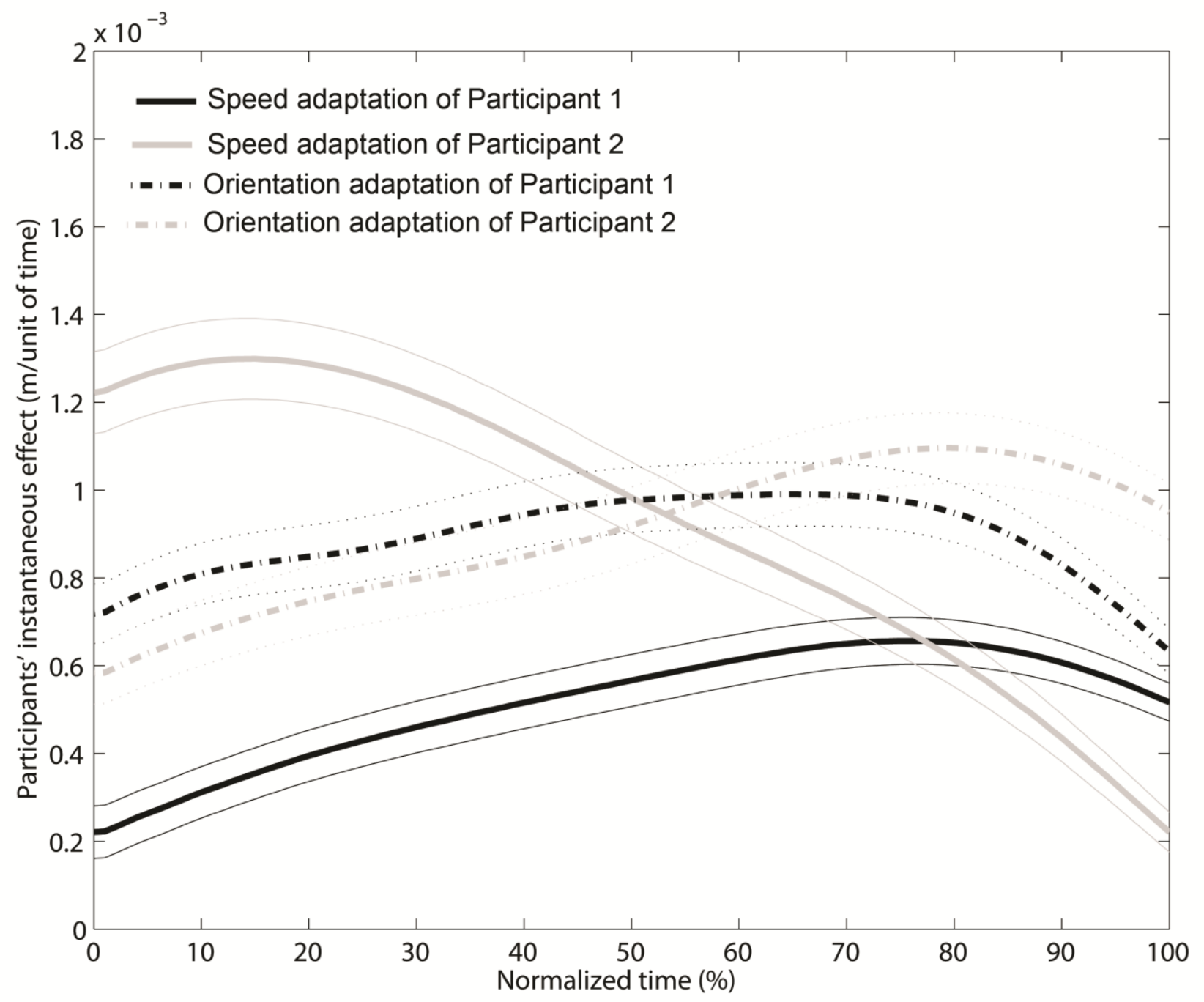

